\title{
Test of QCD analytic predictions for the multiplicity ratio between gluon and quark jets
}

\section{OPAL Collaboration}

G. Alexander ${ }^{\mathrm{w}}$, J. Allison ${ }^{\mathrm{p}}$, N. Altekamp ${ }^{\mathrm{e}}$, K. Ametewee ${ }^{\mathrm{y}}$, K.J. Anderson ${ }^{\mathrm{i}}$, S. Anderson ${ }^{\ell}$, S. Arcelli ${ }^{\text {b }}$, S. Asai ${ }^{x}$, D. Axen ${ }^{\text {ac }}$, G. Azuelos ${ }^{\text {r,1 }}$, A.H. Ball ${ }^{\mathrm{q}}$, E. Barberio ${ }^{\text {h }}$, R.J. Barlow ${ }^{\text {p }}$ R. Bartoldus ${ }^{\text {c }}$, J.R. Batley ${ }^{\mathrm{e}}$, J. Bechtluft ${ }^{\mathrm{n}}$, C. Beeston $^{\mathrm{p}}$, T. Behnke ${ }^{\mathrm{h}}$, A.N. Bell ${ }^{\mathrm{a}}$, K.W. Bell ${ }^{\mathrm{t}}$, G. Bella ${ }^{\mathrm{w}}$, S. Bentvelsen ${ }^{\mathrm{h}}$, P. Berlich $^{\mathrm{j}}$, S. Bethke $^{\mathrm{n}}$, O. Biebel ${ }^{\mathrm{n}}$, V. Blobel ${ }^{\mathrm{h}}$, I.J. Bloodworth ${ }^{\mathrm{a}}$, J.E. Bloomer ${ }^{\mathrm{a}}$, M. Bobinski ${ }^{\mathrm{j}}$, P. Bock ${ }^{\mathrm{k}}$, H.M. Bosch ${ }^{\mathrm{k}}$, M. Boutemeur ${ }^{\text {ah }}$, B.T. Bouwens ${ }^{\ell}$, S. Braibant ${ }^{\ell}$, R.M. Brown ${ }^{\text {t }}$, H.J. Burckhart ${ }^{\text {h}}$, C. Burgard ${ }^{\text {h }}$, R. Bürgin ${ }^{\mathrm{j}}$, P. Capiluppi ${ }^{b}$, R.K. Carnegie ${ }^{f}$, A.A. Carter ${ }^{\mathrm{m}}$, J.R. Carter ${ }^{\mathrm{e}}$, C.Y. Chang ${ }^{\mathrm{q}}$, C. Charlesworth ${ }^{\mathrm{f}}$, D.G. Charlton ${ }^{\mathrm{a}, 2}$, D. Chrisman ${ }^{\mathrm{d}}$, S.L. Chu ${ }^{\mathrm{d}}$, P.E.L. Clarke ${ }^{\mathrm{o}}$, I. Cohen ${ }^{\mathrm{w}}$, J.E. Conboy ${ }^{\mathrm{o}}$, O.C. Cooke $^{\mathrm{p}}$, M. Cuffiani ${ }^{\mathrm{b}}$, S. Dado ${ }^{\mathrm{v}}$, C. Dallapiccola ${ }^{\mathrm{q}}$, G.M. Dallavalle ${ }^{\mathrm{b}}$, S. De Jong ${ }^{\ell}$, L.A. del Pozo ${ }^{h}$, K. Desch ${ }^{c}$, M.S. Dixit ${ }^{g}$, E. do Couto e Silva ${ }^{\ell}$, M. Doucet ${ }^{\mathrm{r}}$, E. Duchovni ${ }^{\mathrm{z}}$, G. Duckeck ${ }^{\text {ah }}$, I.P. Duerdoth ${ }^{p}$, J.E.G. Edwards ${ }^{\text {p }}$, P.G. Estabrooks ${ }^{f}$, H.G. Evans ${ }^{i}$, M. Evans ${ }^{\mathrm{m}}$, F. Fabbri ${ }^{\mathrm{b}}$, P. Fath ${ }^{\mathrm{k}}$, F. Fiedler ${ }^{\ell}$, M. Fierro ${ }^{\mathrm{b}}$, H.M. Fischer ${ }^{\mathrm{c}}$, R. Folman ${ }^{\mathrm{z}}$, D.G. Fong ${ }^{\mathrm{q}}$, M. Foucher ${ }^{\mathrm{q}}$, A. Fürtjes ${ }^{\mathrm{h}}$, P. Gagnon ${ }^{\mathrm{g}}$, A. Gaidot ${ }^{\mathrm{u}}$, J.W. Gary ${ }^{\mathrm{d}}$, J. Gascon ${ }^{\mathrm{r}}$, S.M. Gascon-Shotkin ${ }^{q}$, N.I. Geddes ${ }^{t}$, C. Geich-Gimbel ${ }^{c}$, F.X. Gentit ${ }^{u}$, T. Geralis ${ }^{t}$, G. Giacomelli ${ }^{\text {, }}$, P. Giacomelli ${ }^{\mathrm{d}}$, R. Giacomelli ${ }^{\mathrm{b}}$, V. Gibson ${ }^{\mathrm{e}}$, W.R. Gibson ${ }^{\mathrm{m}}$, D.M. Gingrich ad,1, D. Glenzinski ${ }^{i}$, J. Goldberg ${ }^{v}$, M.J. Goodrick ${ }^{\mathrm{e}}$,W. Gorn ${ }^{\mathrm{d}}$, C. Grandi ${ }^{\mathrm{b}}$, E. Gross ${ }^{\mathrm{z}}$, M. Gruwé ${ }^{\mathrm{h}}$, C. Hajdu ${ }^{\text {af }}$, G.G. Hanson ${ }^{\ell}$, M. Hansroul ${ }^{\text {h }}$, M. Hapke ${ }^{\mathrm{m}}$, C.K. Hargrove ${ }^{\text {g, P.A. Hart }}{ }^{\mathrm{i}}$, C. Hartmann ${ }^{\mathrm{c}}$, M. Hauschild ${ }^{\mathrm{h}}$, C.M. Hawkes ${ }^{\mathrm{e}}$, R. Hawkings ${ }^{\text {h }}$, R.J. Hemingway ${ }^{\text {f }}$, G. Herten ${ }^{j}$, R.D. Heuer ${ }^{\text {h }}$, M.D. Hildreth ${ }^{\text {h }}$, J.C. Hill ${ }^{\text {e }}$, S.J. Hillier ${ }^{a}$, T. Hilse ${ }^{\mathrm{j}}$, P.R. Hobson ${ }^{\mathrm{y}}$, R.J. Homer ${ }^{\mathrm{a}}$, A.K. Honma ${ }^{\mathrm{ab}, 1}$, D. Horváth ${ }^{\mathrm{af}, 3}$, R. Howard ${ }^{\mathrm{ac}}$, R.E. Hughes-Jones ${ }^{p}$, D.E. Hutchcroft ${ }^{\mathrm{e}}$, P. Igo-Kemenes ${ }^{\mathrm{k}}$, D.C. Imrie ${ }^{\mathrm{y}}$, M.R. Ingram ${ }^{\mathrm{p}}$, K. Ishii ${ }^{\mathrm{x}}$, A. Jawahery ${ }^{\mathrm{q}}$, P.W. Jeffreys ${ }^{\mathrm{t}}$, H. Jeremie ${ }^{\mathrm{r}}$, M. Jimack ${ }^{\mathrm{a}}$, A. Joly ${ }^{\mathrm{r}}$, C.R. Jones ${ }^{\mathrm{e}}$, G. Jones ${ }^{p}$, M. Jones ${ }^{f}$, R.W.L. Jones ${ }^{h}$, U. Jost ${ }^{k}$, P. Jovanovic ${ }^{a}$, T.R. Junk ${ }^{h}$, D. Karlen ${ }^{f}$, K. Kawagoe ${ }^{\text {x }}$, T. Kawamoto ${ }^{\text {x }}$, R.K. Keeler ${ }^{\text {ab }}$, R.G. Kellogg ${ }^{q}$, B.W. Kennedy ${ }^{\text {t }}$, B.J. King ${ }^{\text {h }}$, J. Kirk ${ }^{\text {ac }}$, S. Kluth ${ }^{\text {h }}$, T. Kobayashi ${ }^{x}$, M. Kobel ${ }^{j}$, D.S. Koetke ${ }^{\text {f }}$, T.P. Kokott ${ }^{c}$, M. Kolrep ${ }^{j}$, S. Komamiya ${ }^{x}$, R. Kowalewski ${ }^{\text {h}}$, T. Kress ${ }^{k}$, P. Krieger ${ }^{\text {f }}$, J. von Krogh ${ }^{k}$, P. Kyberd ${ }^{\text {m }}$, G.D. Lafferty ${ }^{\mathrm{p}}$, H. Lafoux ${ }^{\mathrm{u}}$, R. Lahmann ${ }^{\mathrm{q}}$, W.P. Lai ${ }^{\text {s }}$, D. Lanske ${ }^{\mathrm{n}}$, J. Lauber ${ }^{\mathrm{o}}$, S.R. Lautenschlager ${ }^{\text {ae }}$, J.G. Layter ${ }^{\mathrm{d}}$, D. Lazic ${ }^{\mathrm{v}}$, A.M. Lee ${ }^{\text {ae }}$, E. Lefebvre ${ }^{\mathrm{r}}$, D. Lellouch ${ }^{\mathrm{z}}$, 
J. Letts ${ }^{b}$, L. Levinson ${ }^{z}$, C. Lewis ${ }^{\text {o }}$, S.L. Lloyd ${ }^{\mathrm{m}}$, F.K. Loebinger ${ }^{\mathrm{p}}$, G.D. Long ${ }^{\mathrm{q}}$, M.J. Lostyg, J. Ludwig ${ }^{i}$, A. Malik ", M. Mannclli ${ }^{\text {h }}$, S. Marcellini ${ }^{\text {b }}$, C. Markus ${ }^{\mathrm{c}}$, A.J. Martin ${ }^{\mathrm{m}}$, J.P. Martin ${ }^{\mathrm{r}}$, G. Martinez ${ }^{\mathrm{q}}$, T. Mashimo ${ }^{\mathrm{x}}$, W. Matthews ${ }^{\mathrm{y}}$, P. Mättig ${ }^{\mathrm{c}}$, W.J. McDonald ${ }^{\text {ad }}$, J. McKenna ${ }^{\text {ac }}$, E.A. Mckigney ${ }^{\circ}$, T.J. McMahon ${ }^{a}$, A.I. McNab ${ }^{\mathrm{m}}$, R.A. McPherson ${ }^{\text {h }}$, F. Meijers ${ }^{h}$, S. Menke ${ }^{\text {c }}$, F.S. Merritt ${ }^{i}$, H. Mes ${ }^{g}$, J. Meyer ${ }^{\text {aa }}$, A. Michelini ${ }^{\text {b }}$, G. Mikenberg ${ }^{z}$, D.J. Miller ${ }^{\mathrm{o}}$, R. Mir ${ }^{\mathrm{z}}$, W. Mohr ${ }^{\mathrm{j}}$, A. Montanari ${ }^{\mathrm{b}}$, T. Mori $^{\mathrm{x}}$, M. Morii ${ }^{x}$, U. Müller ${ }^{c}$, K. Nagai $^{z}$, I. Nakamura ${ }^{x}$, H.A. Neal ${ }^{\text {h }}$, B. Nellen ${ }^{\text {c }}$, B. Nijjhar ${ }^{\text {, }}$, R. Nisius ${ }^{h}$, S.W. O'Neale ${ }^{\text {a }}$, F.G. Oakham ${ }^{g}$, F. Odorici ${ }^{b}$, H.O. Ogren ${ }^{\ell}$, T. Omori ${ }^{x}$, M.J. Oreglia ${ }^{i}$, S. Orito ${ }^{x}$, J. Pálinkás ${ }^{\text {ag, }}{ }^{4}$, G. Pásztor ${ }^{\text {af }}$, J.R. Pater ${ }^{p}$, G.N. Patrick ${ }^{t}$, J. Patt ${ }^{j}$, M.J. Pearce ${ }^{\text {a }}$, S. Petzold ${ }^{\text {aa }}$, P. Pfeifenschneider ${ }^{n}$, J.E. Pilcher ${ }^{\mathrm{i}}$, J. Pinfold ${ }^{\text {ad }}$, D.E. Plane ${ }^{\mathrm{h}}$, P. Poffenberger ${ }^{\text {ab }}$, B. Poli ${ }^{b}$, A. Posthaus ${ }^{c}$, H. Przysiezniak ${ }^{\text {ad }}$, D.L. Rees ${ }^{a}$, D. Rigby ${ }^{a}$, S.A. Robins ${ }^{\mathrm{m}}$, N. Rodning ${ }^{\text {ad }}$, J.M. Roney ${ }^{\mathrm{ab}}$, A. Rooke ${ }^{\mathrm{o}}$, E. Ros ${ }^{\mathrm{h}}$, A.M. Rossi ${ }^{\mathrm{b}}$, M. Rosvick ${ }^{\text {ab }}$, P. Routenburg ${ }^{\text {ad }}$, Y. Rozen ${ }^{\mathrm{v}}$, K. Runge ${ }^{\mathrm{j}}$, O. Runolfsson $^{\mathrm{h}}$, U. Ruppel ${ }^{\mathrm{n}}$, D.R. Rust ${ }^{\ell}$, R. Rylko ${ }^{y}$, K. Sachs ${ }^{j}$, E.K.G. Sarkisyan ${ }^{w}$, M. Sasaki ${ }^{\mathrm{x}}$, C. Sbarra ${ }^{\mathrm{b}}$, A.D. Schaile ${ }^{\text {ah }}$, O. Schaile ${ }^{\text {ah }}$, F. Scharf ${ }^{c}$, P. Scharff-Hansen ${ }^{\text {h }}$, P. Schenk ${ }^{\text {d }}$, B. Schmitt ${ }^{\text {h }}$, S. Schmitt ${ }^{k}$, M. Schröder ${ }^{\text {h }}$, H.C. Schultz-Coulon ${ }^{j}$, M. Schulz ${ }^{\text {h }}$, M. Schumacher ${ }^{\text {c }}$, P. Schütz ${ }^{c}$, W.G. Scott ${ }^{t}$, T.G. Shears ${ }^{p}$, B.C. Shen ${ }^{d}$, C.H. Shepherd-Themistocleous ${ }^{\text {aa }}$, P. Sherwood ${ }^{\circ}$, G.P. Siroli ${ }^{\text {b }}$ A. Sittler ${ }^{\text {aa }}$, A. Skillman ${ }^{\text {, }}$, A. Skuja ${ }^{\mathrm{q}}$, A.M. Smith ${ }^{\mathrm{h}}$, T.J. Smith ${ }^{\mathrm{ab}}$, G.A. Snow ${ }^{\mathrm{q}}$, R. Sobie ${ }^{\mathrm{ab}}$, S. Söldner-Rembold ${ }^{\mathrm{j}}$, R.W. Springer ${ }^{\text {ad }}$, M. Sproston ${ }^{\mathrm{t}}$, A. Stahl ${ }^{\mathrm{c}}$, M. Starks ${ }^{\ell}$, M. Steiert ${ }^{\mathrm{k}}$, K. Stephens ${ }^{\mathrm{p}}$, J. Steuerer ${ }^{\mathrm{aa}}$, B. Stockhausen ${ }^{\text {c }}$, D. Strom ${ }^{s}$, F. Strumia ${ }^{h}$, P. Szymanski ${ }^{t}$, R. Tafirout ${ }^{r}$, S.D. Talbot ${ }^{a}$, S. Tanaka ${ }^{\mathrm{x}}$, P. $\operatorname{Taras}^{\mathrm{r}}$, S. Tarem ${ }^{\mathrm{v}}$, M. Tecchio ${ }^{\mathrm{h}}$, M. Thiergen ${ }^{\mathrm{j}}$, M.A. Thomson ${ }^{\mathrm{h}}$, E. von Törne ${ }^{\mathrm{c}}$, S. Towers ${ }^{\mathrm{f}}$, T. Tsukamoto ${ }^{\mathrm{x}}$, E. Tsur ${ }^{\mathrm{w}}$, A.S. Turcot ${ }^{\mathrm{i}}$, M.F. Turner-Watson ${ }^{\mathrm{h}}$, P. Utzat ${ }^{k}$, R. Van Kooten ${ }^{\ell}$, G. Vasseur ${ }^{\mathrm{u}}$, M. Verzocchi ${ }^{\mathrm{j}}$, P. Vikas ${ }^{\mathrm{r}}$, M. Vincter ${ }^{\mathrm{ab}}$, E.H. Vokurka ${ }^{\text {p }}$, F. Wäckerle ${ }^{\mathrm{j}}$, A. Wagner ${ }^{a}$, C.P. Ward ${ }^{\mathrm{e}}$, D.R. Ward ${ }^{\mathrm{e}}$, J.J. Ward ${ }^{\mathrm{o}}$, P.M. Watkins ${ }^{\text {a }}$, A.T. Watson ${ }^{a}$, N.K. Watson ${ }^{g}$, P. Weber ${ }^{\text {f }}$, P.S. Wells ${ }^{\text {h }}$, N. Wermes ${ }^{c}$, J.S. White ${ }^{\mathrm{ab}}$, B. Wilkens ${ }^{\mathrm{j}}$, G.W. Wilson ${ }^{\mathrm{aa}}$, J.A. Wilson ${ }^{\mathrm{a}}$, G. Wolf ${ }^{\mathrm{z}}$, S. Wotton ${ }^{\mathrm{e}}$, T.R. Wyatt ${ }^{p}$, S. Yamashita ${ }^{x}$, G. Yekutieli ${ }^{z}$, V. Zacek $^{r}$

a School of Physics and Space Research, University of Birmingham, Birmingham B15 2TT, UK

b Dipartimento di Fisica dell' Università di Bologna and INFN, I-40126 Bologna, Italy

c Physikalisches Institut, Universität Bonn, D-53115 Bonn, Germany

${ }^{d}$ Department of Physics, University of California, Riverside CA 92521, USA e Cavendish Laboratory, Cambridge CB3 OHE, UK

f Ottawa-Carleton Institute for Physics, Department of Physics, Carleton University, Ottawa, Ontario K1S 5B6, Canada g Centre for Research in Particle Physics, Carleton University, Ottawa, Ontario K1S 5B6, Canada

${ }^{\text {h }}$ CERN, European Organisation for Particle Physics, CH-1211 Geneva 23, Switzerland

i Enrico Fermi Institute and Department of Physics, University of Chicago, Chicago IL 60637, USA

j Fakultät für Physik, Albert Ludwigs Universität, D-79104 Freiburg, Germany

${ }^{\mathrm{k}}$ Physikalisches Institut, Universität Heidelberg, D-69120 Heidelberg, Germany

${ }^{\ell}$ Indiana University, Department of Physics, Swain Hall West 117, Bloomington IN 47405, USA

${ }^{m}$ Queen Mary and Westfield College, University of London, London EI 4NS, UK

n Technische Hochschule Aachen. III Physikalisches Institut, Sommerfeldstrasse 26-28, D-52056 Aachen, Germany

- University College London, London WCIE 6BT, UK

p Department of Physics, Schuster Laboratory, The University, Manchester M13 9PL, UK q Department of Physics, University of Maryland, College Park, MD 20742, USA 


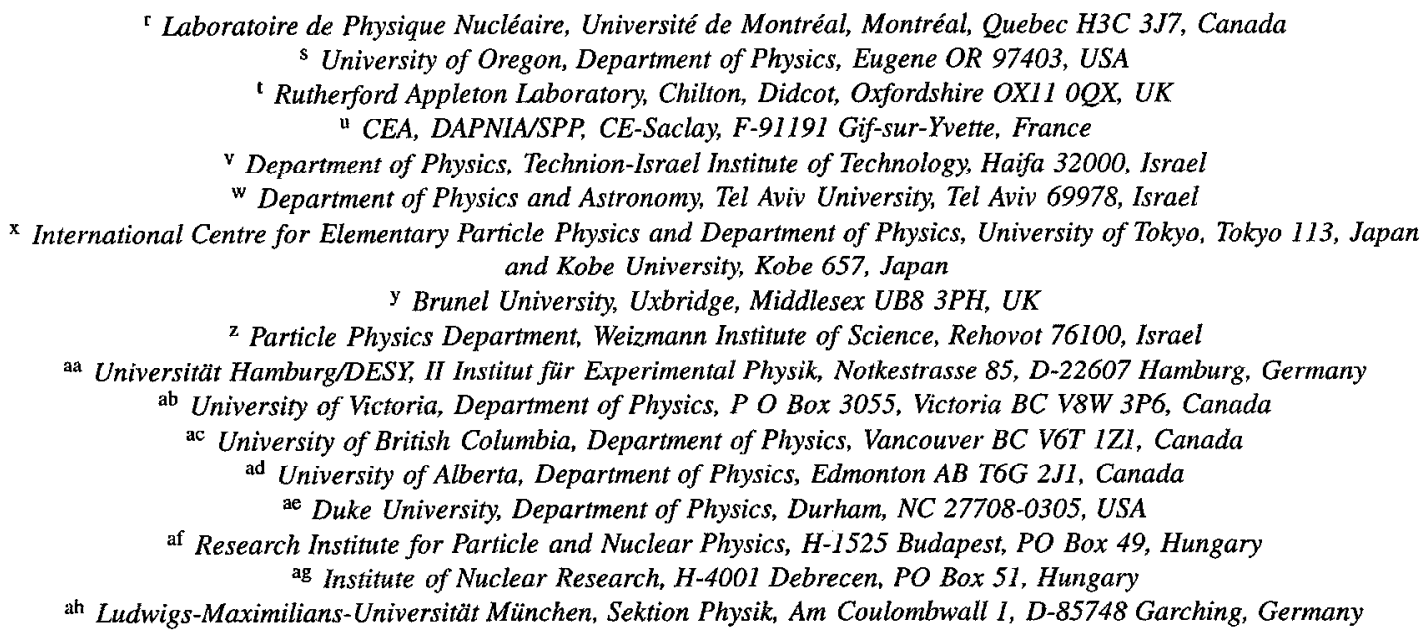

Received 9 August 1996; revised manuscript received 24 September 1996

Editor: K. Winter

\begin{abstract}
Gluon jets with about $39 \mathrm{GeV}$ energy are identified in hadronic $Z^{0}$ decays by tagging two jets in the same hemisphere of an event as quark jets. Identifying the gluon jet to be all the particles observed in the hemisphere opposite to that containing the two tagged jets yields an inclusive gluon jet definition corresponding to that used in analytic calculations, allowing the first direct test of those calculations. In particular, this jet definition yields results which are only weakly dependent on a jet finding algorithm. We find

$r_{\text {ch. }}=1.552 \pm 0.041$ (stat.) \pm 0.061 (syst.)

for the ratio of the mean charged particle multiplicity in gluon jets to that in light quark uds jets, where the uds jets are identified using an inclusive jet definition similar to that used for the gluon jets. Our result is in general agreement with the prediction of a recent analytic calculation which incorporates energy conservation into the parton shower branching processes, but is considerably smaller than analytic predictions which do not incorporate energy conservation.
\end{abstract}

\section{Introduction}

Quantum Chromodynamics (QCD) predicts differences between the properties of jets initiated by gluons and those initiated by quarks. These differences are due to the different relative probabilities for a gluon or a quark to radiate an additional gluon, given by the Casimir terms $C_{A}=3$ and $C_{F}=4 / 3$, respectively. In

\footnotetext{
${ }^{1}$ And at TRIUMF, Vancouver, Canada V6T $2 \mathrm{~A} 3$

2 And Royal Society University Research Fellow

3 And Institute of Nuclear Research, Debrecen, Hungary

${ }^{4}$ And Department of Experimental Physics, Lajos Kossuth University, Debrecen, Hungary
}

particular, QCD analytic predictions exist for the ratio between the mean particle multiplicity of gluon and quark jets [1-3]. Clear differences between gluon and quark jets have been established experimentally. The main results are from the experiments operating at the LEP $\mathrm{e}^{+} \mathrm{e}^{-}$storage ring at CERN [4-10]. The differences observed are in qualitative agreement with the theoretical predictions: gluon jets are measured to have a larger mean particle multiplicity, a softer fragmentation function and a larger angular width than quark jets [11]. Direct quantitative tests of the analytic results have not yet been possible, however, because of differences between the thcorctical and experimental 
definitions of the jets and event samples. The LEP experiments have so far relied on mathematical jet finding algorithms to select exclusive samples of three-jet events. Those same algorithms have also been used to assign the particles in an event to the quark and gluon jets under study. The results have been shown to depend on the algorithm chosen $[6,8]$. In contrast, the analytic calculations employ definitions of the event samples and jets which are entirely inclusive. For the calculations, two samples of events are chosen: a sample of gluon-gluon gg events produced from a color singlet point source is used to define the gluon jet properties and a sample of quark-antiquark $q \bar{q}$ events produced under the same circumstances is used to define the quark jet properties. The gluon and quark jet characteristics are given by inclusive sums over the particles in these two samples. Thus, the theoretical results are not restricted to three-jet events and do not employ a jet finder to assign particles to the jets.

The experimental difficulty in obtaining a jet definition corresponding to the theoretical one lies in the gluon jet sample, since gg production from a point source does not occur naturally in $\mathrm{e}^{+} \mathrm{e}^{-}$annihilations. In contrast, the $\mathrm{q} \overrightarrow{\mathrm{q}}$ sample employed by the theory is the inclusive $\mathrm{e}^{+} \mathrm{e}^{-}$multihadronic one and so is readily available. In [12], a method was proposed for LEP experiments to identify gluon jets using an inclusive definition similar to that used for the analytic calculations. The method is based on rare events of the type $\mathrm{e}^{+} \mathrm{e}^{-} \rightarrow \mathrm{q} \overline{\mathrm{q}} \mathrm{g}_{\text {incl. }}$ in which the $\mathrm{q}$ and $\overline{\mathrm{q}}$ are identified quark jets which appear in the same hemisphere of an event. The quantity $g_{\text {incl, }}$ taken to be the gluon jet, is defined by the sum of all particles observed in the hemisphere opposite to that containing the $q$ and $\bar{q}$. In the limit that the $\mathrm{q}$ and $\overline{\mathrm{q}}$ are collinear, the gluon jet $\mathrm{g}_{\text {incl. }}$ is produced under the same conditions as the gluon jets in gg events from a color singlet point source. The jets $g_{\text {incl. }}$ therefore correspond closely to single gluon jets in gg events, defined by dividing the gg events in half using the plane perpendicular to the principal event axis.

In this paper, we present the results of a study based on this gluon jet identification method, performed using the data sample of the OPAL detector at LEP. One change we implement rclative to the suggestions in [12] is that we select light quark uds events for the quark jet sample rather than inclusive multihadronic events. As a consequence, we obtain a better corre- spondence between the data and the massless quark assumption used for the analytic formulae. The experimental result is compared to QCD analytic predictions to yield the first direct quantitative test of them. The result is also compared to the predictions of Monte Carlo simulation programs which incorporate perturbative QCD and models for hadronization.

\section{Detector and data sample}

The OPAL detector is described in detail elsewhere [13]. The present analysis is based on a sample of about 3712000 hadronic events collected by OPAL from 1991 to 1995 . Charged tracks and clusters of energy measured in the electromagnetic calorimeter were selected for the analysis using the criteria given in [7]. To minimize double counting of energy, clusters were used only if they were not associated with a charged track. Each accepted track and unassociated cluster was considered to be a particle. Tracks were assigned the pion mass. Clusters were assigned zero mass since they originate mostly from photons. To eliminate residual background and events in which a significant number of particles was lust near the beam direction, the number of accepted charged tracks was required to be at least five and the thrust axis [14] of the event, calculated using the particles, was required to satisfy $\left|\cos \left(\theta_{\text {thrust }}\right)\right|<0.9$, where $\theta_{\text {thrust }}$ is the angle between the thrust and beam axes. The residual background from all sources was estimated to be less than $1 \%$.

\section{Gluon jet selection}

For our study, a gluon jet is defined inclusively by the particles observed in the hemisphere opposite to that containing an identified quark and antiquark jet, as stated in the introduction. To select this gluon jet sample, each event is divided into hemispheres using the plane perpendicular to the thrust axis. Exactly two jets are reconstructed in each hemisphere, using a jet finder.

We choose the $k_{\perp}$ ("Durham") jet finder [15] because this is a standard choice for $Z^{0}$ physics. The results for the gluon jet properties are only weakly sensitive to this choice, as discussed in [12] and below 
in Section 8. Next, we attempt to reconstruct a displaced secondary vertex in each of the four jets. Displaced secondary vertices are associated with heavy quark decay, especially that of the b quark. At LEP, b quarks are produced almost exclusively at the electroweak vertex: thus a jet containing a $b$ hadron is almost always a quark jet. To identify secondary vertices in jets, we employ the method given in [6]. Briefly, a secondary vertex is required to contain at least three tracks, at least two of which are "significant". A track is significant if its signed impact parameter value in the $r-\phi$ plane $^{5}$ with respect to the primary event vertex, $b$, satisfies $b / \sigma_{b}>2.5$, with $\sigma_{b}$ the error of $b$. For jets with such a secondary vertex, the signed decay length, $L$, is calculated with respect to the primary vertex, along with its error, $\sigma_{L}$. To be tagged as a quark jet, a jet is required to have a visible energy of at least $5 \mathrm{GeV}$ and a successfully reconstructed secondary vertex with a decay length significance, given by $L / \sigma_{L}$, greater than 5.0 . The visible energy of a jet is defined by the sum of the cncrgy of the particles assigned to the jet. Events are selected if both jets in one of the two hemispheres are tagged: 1829 such events are selected. Of these, one event has two tagged jets in both hemispheres: both hemispheres of this event are eliminated by the cuts described below. We refer to a hemisphere with two tagged jets as a tagged hemisphere.

We next examine the angles that the two jets in the tagged hemisphere make with respect to the thrust axis and to each other. These distributions are shown in Fig. 1. In this figure, "jet a" refers to the higher energy of the two jets in the tagged hemisphere and "jet b" to the lower energy of the two jets. The corresponding predictions from the Jetset parton shower Monte Carlo [16] are also shown. The Jetset results include simulation of the OPAL detector [17] and the same analysis procedures as are applied to the data. The Jetset sample is a combination of events generated using version 7.3 of the program with the parameter values given in [5] and of events generated using version 7.4 of the program with the parameter values given in [7]. The initial Monte Carlo samples have about 3000000

\footnotetext{
${ }^{5}$ Our coordinate system is defined so that $z$ is the coordinate parallel to the $e^{-}$beam axis, $r$ is the coordinate normal to the beam axis, $\phi$ is the azimuthal angle around the beam axis and $\theta$ is the polar angle with respect to $z$.
}
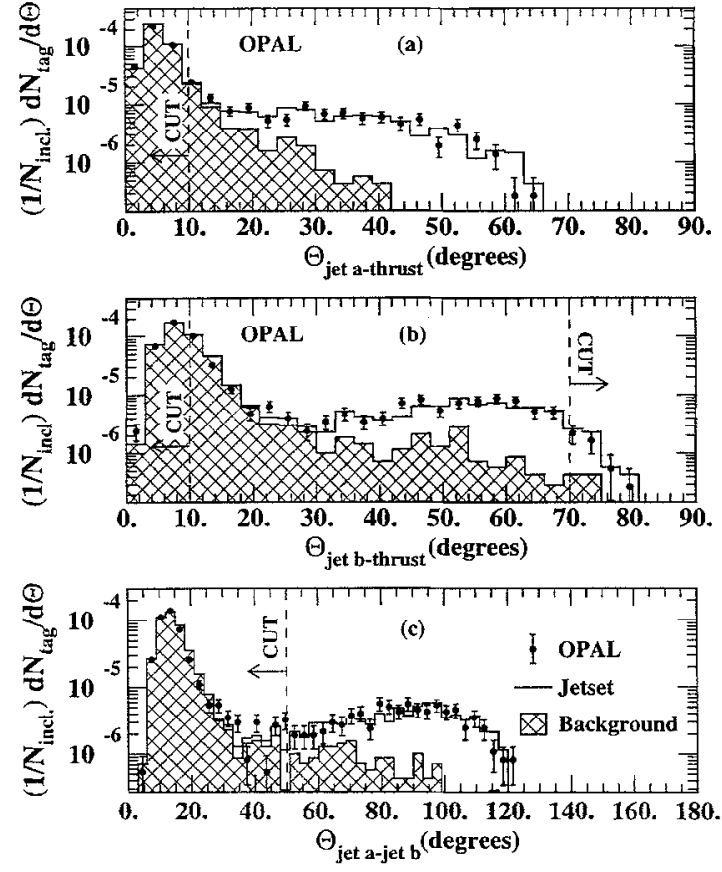

Fig. 1. (a) Angle hetween the thrust axis and jet $a$, where jet a is the higher energy of the two jets in event hemispheres with two tagged quark jets; (b) corresponding distribution for jet $b$, which is the lower energy of the two jets; (c) angle between jet a and jet $b$. The distributions are shown for the uncorrected data and for the Jetset Monte Carlo with detector simulation.

events for version 7.3 and 4000000 events for version 7.4. The two Jetset versions yield results which are consistent with each other to within the statistical uncertainties and so we combine them. The estimates of the gluon and quark jet purities given in this paper are based upon this combined sample. The distributions in Fig. 1 are normalized by the total number of events in the inclusive multihadronic samples, $\mathrm{N}_{\text {incl, }}$, rather than by the number of entries in the histograms, $\mathrm{N}_{\text {tag }}$. The overall description of the data by the Monte Carlo is seen to be good. This agreement between the data and Monte Carlo establishes that the absolute tag rate, defined by $\mathrm{N}_{\text {tag }} / \mathrm{N}_{\text {incl. }}$, is well simulated. The tag rate is $(4.93 \pm 0.11$ (stat.) $) \times 10^{-4}$ for the data and $(5.11 \pm 0.09$ (stat.) $) \times 10^{-4}$ for the Monte Carlo. After the final cuts to select the $g_{\text {incl. }}$ sample, described below, these numbers become $(7.49 \pm 0.45$ (stat.) $) \times$ $10^{-5}$ and $(7.10 \pm 0.32$ (stat.) $) \times 10^{-5}$, respectively.

Using the Jetset events, the hadron lcvel jets are examined to determine whether they are associated with 
an underlying quark or antiquark jet. To perform this association, the Monte Carlo events are also examined at the parton level. The two hadron jets (of the four which are reconstructed) closest in angle to the directions of the primary quark and antiquark which have evolved from the $Z^{0}$ decay are considered to be quark (or antiquark) jets. The contributions to the Monte Carlo results from events in which at least one of the jets in the tagged hemisphere is not identified as a quark (or antiquark) jet are shown by the crosshatched regions in Fig. 1: these events represent background for our study.

From Figs. 1(a) and (b), it is seen that the background is concentrated at small values of angle between the jets and thrust axis. From Fig. 1(c), it is seen that the background is also concentrated at small values of angle between the two jets. If two jets are close together, or if one of the two jets is much more energetic than the other, it is very likely that one of the two jets is a gluon jet as a consequence of the gluon radiation matrix element. In our previous study of symmetric events [6], in which two well separated jets with energies of about $24 \mathrm{GeV}$ were present in the same hemisphere of an event, with one of the jets a quark jet and the other almost always a gluon jet, our secondary vertex tagging method was able to distinguish the gluon jet from the quark jet with about $93 \%$ purity. It is much more difficult to identify two quark jets in the same hemisphere if the kinematic configuration is similar to that of gluon radiation from a quark, however (i.e. if one of the jets is collinear or soft in comparison to the other jet). This explains the distribution of the background in Fig. 1.

To reduce the background, we require the angle between the jets and thrust axis to exceed $10^{\circ}$ and the angle between the two jets to exceed $50^{\circ}$. A last requirement is that the two jets lie no more than $70^{\circ}$ from the thrust axis in order to eliminate jets near the hemisphere boundary. In total, 278 events are selected

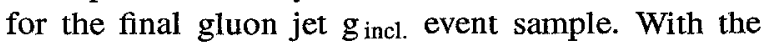
final cuts, Jetset predicts that both jets in the tagged hemisphere are quark jets with $(83.0 \pm 1.7) \%$ probability, where the uncertainty is statistical: this is the estimated purity of the $g_{\text {incl. }}$ gluon jet sample.

The mean energy of the gluon jets, $\langle E\rangle_{\mathrm{g} \text { ick. }}$, is less than the beam energy because the two quark jets against which $g_{\text {incl. }}$ recoils are not entirely collinear. To determine the gluon jet energy, we use the method of calculated jet energies. A jet direction is determined for the gluon jet by summing the momenta of the particles in the $\mathrm{g}_{\text {incl. }}$ hemisphere. This jet direction is used in conjunction with those of the two jets in the tagged hemisphere to calculate the jet energies assuming massless kinematics and perfect event reconstruction. ${ }^{6}$ The energies calculated from the jet directions are known to be more reliable than the visible energies because they are less sensitive to detector inefficiencies, assignments of particles to jets, and variations in the particle selection criteria. Using this method, the mean gluon jet energy is determined to be $\langle E\rangle_{\mathrm{g}_{\text {incl. }}}=39.2 \pm 0.3$ (stat.) \pm 1.8 (syst.) $\mathrm{GeV}$. This value includes a multiplicative correction of 1.02 to account for the effects of detector response and initial-state photon radiation. The method in which the correction factor is obtained is presented in Section 5 . The systematic error assigned to $\langle E\rangle_{\mathrm{g}_{\text {incl. }}}$ is given by the difference between the mean calculated and visible jet energies. The measured visible jet energy has also been corrected using the method discussed in Section 5.

Because we rely on heavy quark tagging to identify quark jets, the $g_{\text {incl. jets in our study are contained }}$ in heavy quark events: the Monte Carlo with detector simulation predicts that $96 \%$ of the events in the $g_{\text {incl. }}$. sample are $b$ events. This reliance on $b$ events is not expected to affect our results since the properties of hard, acollinear gluon jets do not depend on the event flavor according to QCD [18]. For example, using the Jetset 7.4 generator with the selection criteria for the $g_{\text {incl. }}$ jets given above, except employing Monte Carlo information to identify quark jets in the manner described above rather than displaced secondary vertices, we find the mean charged particle multiplicity of the $g_{\text {incl. }}$ jets to be the same within the statistical uncertainties for both $b$ and light quark uds events (about 15.0 \pm 0.1 (stat.)). Biases introduced by the heavy quark tagging algorithm are expected to be removed by the correction procedure described in Section 5 because the relevant $b$ jet properties have been shown to be well simulated by the Monte Carlo program [6,7]. Similarly, we do not expect unsimulated aspects of the $b$ hadron production or decay mecha-

\footnotetext{
${ }^{6}$ A study with the Jetset Monte Carlo shows that the finite $b$ quark mass does not result in a significant uncertainty in our determination of the $g_{\text {incl. }}$ jet energy.
} 
nisms to result in measurable biases, because of the good agreement for $b$ jet properties between the data and Monte Carlo found in our earlier studies [6,7].

\section{4. uds quark jet selection}

The uds quark jets in our study are defined inclusively using particles observed in event hemispheres opposite to those containing identified uds jets. Since there are only 278 gluon jets in our study, it is not necessary to use the entire data sample of over 3700000 events mentioned in Section 2 to select the uds jets. Instead, we base this selection on an initial sample of about 836000 hadronic events with center-of-mass (c.m.) energies within $100 \mathrm{MeV}$ of the $Z^{0}$ peak.

To select the uds jet sample, we divide each event into hemispheres using the plane perpendicular to the thrust axis. Selection criteria are applied to each hemisphere separately using charged tracks that appear in a cone of half angle $40^{\circ}$ around the thrust axis. The reason for the restriction to tracks which lie within $40^{\circ}$ of the thrust axis is to avoid using tracks near the hemisphere boundary. The selection criteria are based on the signed impact parameter significance, $b / \sigma_{b}$, and the scaled energy value, $x=2 E / E_{\text {c.m. }}$, of charged tracks which appear in this $40^{\circ}$ cone.

An algorithm is applied to identify charged tracks which are consistent with arising from photon conversions [19]. Removing such tracks from consideration, the number of tracks in the cone which have $b / \sigma_{b}>1.5, N_{\text {sig. }}^{\text {hemis. }}$, is determined. We also determine the maximum $x$ value, $x_{\max }$, of charged tracks in the cone. Because $\mathrm{b}$ and $\mathrm{c}$ hadrons have larger mean decay multiplicities than most hadrons containing only $u, d$ and $\mathrm{s}$ valence quarks, $\mathrm{b}$ and $\mathrm{c}$ jets are less likely to contain charged stable particles with large $x$ values than uds jets. A hemisphere is tagged as containing a uds jet if $N_{\text {sig. }}^{\text {hemis. }}=0$ and $x_{\max }>0.50$. In total, 28007 hemispheres are tagged. This number includes 372 events for which both hemispheres are tagged. The estimated uds purity of the sample, obtained by treating Jetset events with detector simulation in the same manner as the data, is $(93.2 \pm 0.2) \%$, where the uncertainty is statistical. Unlike the gluon jets discussed in Section 3 , the energy of the uds jets is given by the beam energy, $45.6 \mathrm{GeV}$, and has essentially no uncertainty.

\section{Correction method}

To correct the mean energy and multiplicity measurements for detector response and the effects of initial-state photon radiation, correction factors are derived using two different samples of simulated $Z^{0}$ events generated using Jetset. The first sample, based on inclusive $\mathrm{Z}^{0}$ hadronic decays, includes initial-state photon radiation, simulation of the OPAL detector, and the same analysis procedures as the data. The second sample does not include initial-state photon radiation or detector simulation and treats all charged and neutral particles with mean lifetimes greater than $3 \times 10^{-10} \mathrm{~s}$ as stable: hence charged particles from the decays of $\mathrm{K}_{\mathrm{S}}^{0}$ and hyperons are included in the definition of multiplicity. For the correction of the gluon jet data, inclusive $Z^{0}$ events are used for the second sample. The quark jets in this sample are identified with Monte Carlo information using the method discussed in Section 3: otherwise the $g_{\text {incl. }}$ sample is obtained in the same manner as described in Section 3 for the data. For the correction of the uds jet data, the jets of the second sample are defined by the particles in each hemisphere of uds events. Multiplicative correction factors are obtained by taking the ratios of the mean jet energy and multiplicity values predicted by the second sample to those predicted by the first one. Therefore the corrections account not only for detector response and initial-state radiation but also for the background to the $\mathrm{g}_{\text {incl. }}$ and uds jet samples.

The correction factors determined using Jetset 7.3 and Jetset 7.4 with our tuned parameter sets agree with each other within their statistical uncertainties. Therefore, the corrections we apply to the data are obtained by taking the weighted mean of the correction factors derived from the two program versions.

\section{Theoretical predictions}

Before presenting our results, we discuss the theoretical predictions which we wish to test. Various analytic results exist for the ratio $r \equiv\langle n\rangle_{\text {gluon }} /\langle n\rangle_{\text {quark }}$ of the mean number of partons in a gluon jet to that in a quark jet. The original results, valid to leading order, predict $r$ to be $r=\mathrm{C}_{\mathrm{A}} / \mathrm{C}_{\mathrm{F}}=9 / 4$ [1]. Later, higher order corrections valid to the next-to-next-toleading order were found to reduce this result by about 
$10 \%$ [2]. These results do not incorporate energy conservation into the quark and gluon branching processes. Recently, $r$ has been calculated including not only the next-to-next-to-leading order terms but also energy conservation [3], and is found to be reduced yet further in magnitude. Momentum conservation is not included in this latter calculation, however: therefore energy-momentum conservation is only approximate. The analytic results for $r$, valid to leading order (1.o.), to next-to-next to leading order (n.n.l.o.), and including approximate energy-momentum conservation (n.n.l.o., E-cons.) are shown in Fig. 2 as a function of $E_{\text {jet }}=E_{\text {c.m. }} / 2$. For the evaluation of the strong coupling constant, $\alpha_{S}$, the values $n_{f}=5$ and $\Lambda \frac{\left(n_{f}=5\right)}{\mathrm{MS}}=0.209 \mathrm{GeV}$ [20] have been used, where $n_{f}$ is the number of active quark flavors. The results for the two n.n.l.o. calculations are shown as bands: the upper edges of the bands show the results if the energy scale used to evaluate $\alpha_{S}$ is taken to be $E_{\mathrm{c} . \mathrm{m}}$, while the lower edges show the results if this energy scale is taken to be $E_{\text {c.m. }} / 4$. (This choicc of encrgy scales is taken from [21]; the value of $\Lambda_{\frac{\left(n_{f}=5\right)}{\mathrm{MS}}}$ is kept fixed at $0.209 \mathrm{GeV}$ for both choices of the energy scale.) The widths of the bands therefore indicate the level of uncertainty associated with the ambiguity of the energy scale at which to evaluate $\alpha_{S}$.

For our study, the number of active quark flavors $n_{f}$ is the same for the quark and gluon jets, cven though the quark jet sample is restricted to uds events, because $n_{f}$ depends on the number of flavors which participate in the perturbative development of a jet and not on the flavor of the quark and antiquark created in the $Z^{0}$ decay. There is some ambiguity in the value of $n_{f}$ itself, however: in $Z^{0}$ decays, c and b quarks do not participate as actively in the jet evolution as $u$, $\mathrm{d}$ and $\mathrm{s}$ quarks, because of their larger masses. We use the difference between the results obtained using $n_{f}=5$ and $n_{f}=3$ to assess the level of uncertainty in the analytic predictions associated with this ambiguity in $n_{f}$. To evaluate $\alpha_{S}$ for $n_{f}=3$, we use $\Lambda \frac{\left(n_{f}=3\right)}{\mathrm{MS}}=$ $0.340 \mathrm{GeV}$, derived from $\Lambda_{\overline{\mathrm{MS}}}^{\left(n_{f}=5\right)}$ using the prescription relating $\Lambda \frac{\left(n_{f}=3\right)}{\mathrm{MS}}$ to $\Lambda \frac{\left(n_{f}=5\right)}{\mathrm{MS}}$ given in [22]. Essentially the same value of $\Lambda \frac{\left(n_{f}=3\right)}{\mathrm{MS}}$ is derived if the prescription given in [23] is used instead.

Besides the analytic results, a QCD prediction for $r$ can be obtained using Monte Carlo methods: this has

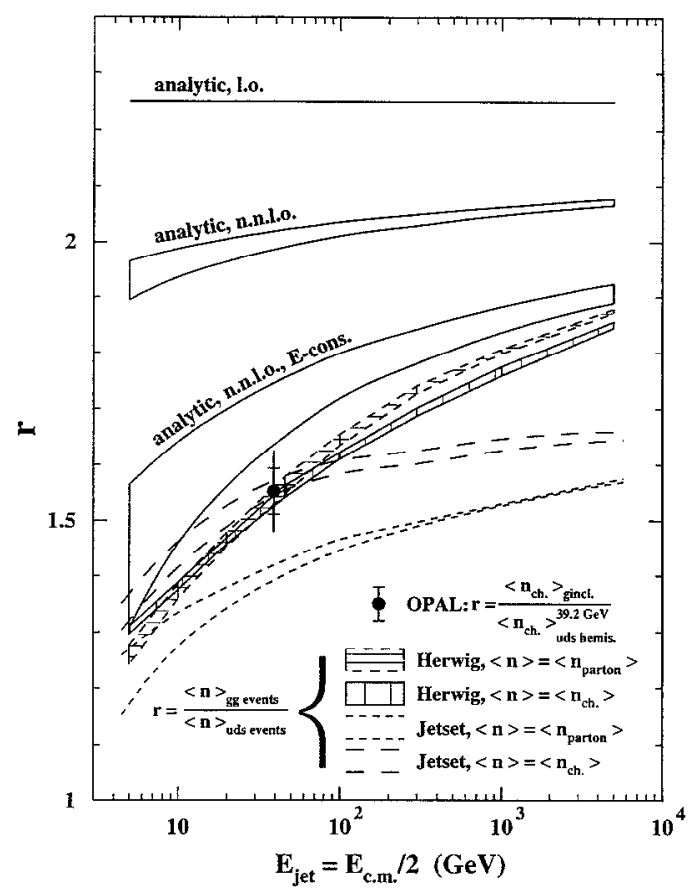

Fig. 2. Analytic and Monte Carlo generator predictions for the ratio of the mean particle multiplicity between gluon and quark jets as a function of jet energy, in comparison to the corrected OPAL measurement for $39 \mathrm{GeV}$ jets. The experimental statistical uncertainty is indicated by the small horizontal bars. The results for the analytic next-to-next to leading order (n.n.l.o.) calculations, evaluated assuming five active quark flavors, are shown as bands. The widths of the bands show the results if the energy scale used to evaluate the strong coupling constant is varied between $E_{\mathrm{c} . \mathrm{m}}$. and $E_{\text {c.m. }} / 4$. The Herwig and Jetset Monte Carlo results for partons and charged hadrons are also shown as bands: the widths of these bands indicate the variation which occurs if the parameters which terminate the parton shower are varied within their allowed ranges.

the advantage that exact energy-momentum conservation is included and that the energy scale used to evaluate $\alpha_{S}$ evolves to correspond to that of each branching. The Herwig parton shower Monte Carlo [24] is particularly suitable for this because -unlike Jetsetit contains perturbative terms beyond leading order. ${ }^{7}$ The parameter values we use for Herwig, version 5.8, are given in [7]. To obtain the Herwig result for $r$, simulated gg events were generated from a color singlet point source in order to correspond to the gg events

\footnotetext{
${ }^{7}$ In addition to the next-to-leading $\sim O\left(\sqrt{\alpha_{S}}\right)$ and next-tonext-to-leading $\sim O\left(\alpha_{S}\right)$ terms included in analytic calculations, Herwig contains terms to order $\sim O\left(\alpha_{S}^{3 / 2}\right)$.
} 
of the analytic calculations. The number of partons present at the end of the perturbative shower, $n_{\text {parton}}$, was determined for each event. The corresponding results from uds $q \bar{q}$ events generated under the same conditions were also determined. The ratio $r$ is defined for the Monte Carlo events by

$r \equiv \frac{\langle n\rangle_{g g \text { events }}}{\langle n\rangle_{\text {uds events }}}$,

with $n=n_{\text {parton. }}$. The resulting Herwig prediction for $r$ is shown by the band with horizontal hatching in Fig. 2. The width of the band shows the variation which occurs if the shower cutoff parameter, VGCUT, is varied between 0.07 and $0.15 \mathrm{GeV}$ from its tuned value of $0.10 \mathrm{GeV}$. This range in the value of VGCUT yields a change in the mean charged particlc multiplicity in inclusive $Z^{0}$ decays of \pm 0.2 units, corresponding to the uncertainty in our measurement of this quantity [25]. The band with vertical hatching in Fig. 2 shows the corresponding result, $r_{\mathrm{ch}}$, obtained by allowing the Herwig events to hadronize and using $n=n_{\text {ch. }}$ in relation (1). It is seen from Fig. 2 that the analytic result which includes energy conservation agrees fairly well with Herwig at both the parton and hadron levels. In contrast, the analytic results without energy conservation do not agree well with Herwig. This suggests that energy-momentum conservation is an essential consideration for the theoretical prediction of $r$. The agreement between Herwig and the analytic results might be improved further if additional higher order terms and exact energy-momentum conservation were included in the latter.

For purposes of comparison, we include in Fig. 2 the corresponding predictions for $r$ from Jetset 7.4, obtained by generating $g g$ and $u d s ~ q \bar{q}$ events as was done for Herwig. For Jetset, the widths of the bands in Fig. 2 arise from varying the shower cutoff parameter, PARJ ( 82), by its uncertainty of $0.50 \mathrm{GeV}$ given in [7] from its tuned value of $1.90 \mathrm{GeV}$ [7]. For the gg events, we use a non-default option ${ }^{8}$ to force the first branching in the parton shower to follow the same first order three-jet matrix element that is imposed on the first branching of the parton shower in the $q \bar{q}$ events [26]. Jetset at the parton and hadron levels is seen to predict significantly lower asymptotic values

\footnotetext{
${ }^{8} \operatorname{PARJ}(47)=4$; this has no effect on the asymptotic behavior of the model predictions.
}

for $r$ at large jet energies than either Herwig or the analytic results.

In some past studies the ratio $r$ has been defined by $r \equiv\left(\langle n\rangle_{\text {gluon }}-1\right) /\left(\langle n\rangle_{\text {quark }}-1\right)$, i.e. by subtracting unity from the numerator and denominator (e.g. see [9]). The motivation for this alternative definition is that the prediction for $r$ is then exactly $\mathrm{C}_{\mathrm{A}} / \mathrm{C}_{\mathrm{F}}=$ $9 / 4$ in the leading logarithm approximation (LLA). However, the LLA includes tree level diagrams only and ignores energy conservation. From Fig. 2, it is seen that higher order terms and energy conservation provide large corrections to $r$ at LEP energies. Thus, there is no strong motivation for this alternative definition of $r$. The analytic results shown in Fig. 2 correspond to $r \equiv\langle n\rangle_{\text {gluon }} /\langle n\rangle_{\text {quark }}$ without the subtraction of unity [27]. Therefore, we adopt this latter definition here.

\section{Results}

The mean charged particle multiplicity value we measure for the gluon jets in our study is $\left\langle n_{\mathrm{ch} .}\right\rangle_{\mathrm{g} \text { ixll }}=$ $14.63 \pm 0.38$ (stat.) \pm 0.59 (syst.). The corresponding result we obtain for the uds jets is $\left\langle n_{\mathrm{ch}}\right\rangle_{\mathrm{udsh}}$ hemis.= $10.05 \pm 0.04$ (stat.) \pm 0.23 (syst.). These values include multiplicative corrections for detector effects and initial-state photon radiation of 1.07 for the gluon jets and 1.02 for the quark jets. The systematic uncertainties for these measurements are discussed in Section 8 . The uds jet result is in agreement with the result $\left\langle n_{\text {ch. }}\right\rangle_{\text {uds hemis. }}=10.41 \pm 0.06$ (stat.) \pm 0.21 (syst.) we found in an earlier publication [28]. This earlier result was obtained by comparing the hemisphere multiplicity in inclusive $Z^{0}$ events with that in tagged c and $b$ events and so is largely uncorrelated with the result presented here.

Our result for $\left\langle n_{\mathrm{ch}}\right\rangle_{\mathrm{g} \text { inc. }}$ is shown in Fig. 3(a). The corresponding results from Herwig and Jetset are shown by the cross and dianond symbols. The Monte Carlo results are obtained using the samples without detector simulation discussed in Section 5 . To illustrate that our definition of gluon jets using $\mathrm{e}^{+} \mathrm{e}^{-}$events corresponds closely to that employed by theory using gg events, we calculate the mean charged particle multiplicity in Herwig and Jetset gg events, $\left\langle n_{\mathrm{ch}}\right\rangle_{\mathrm{gg} \text { events, }}$, and divide the results by two so that they correspond to a single hemisphere: these 


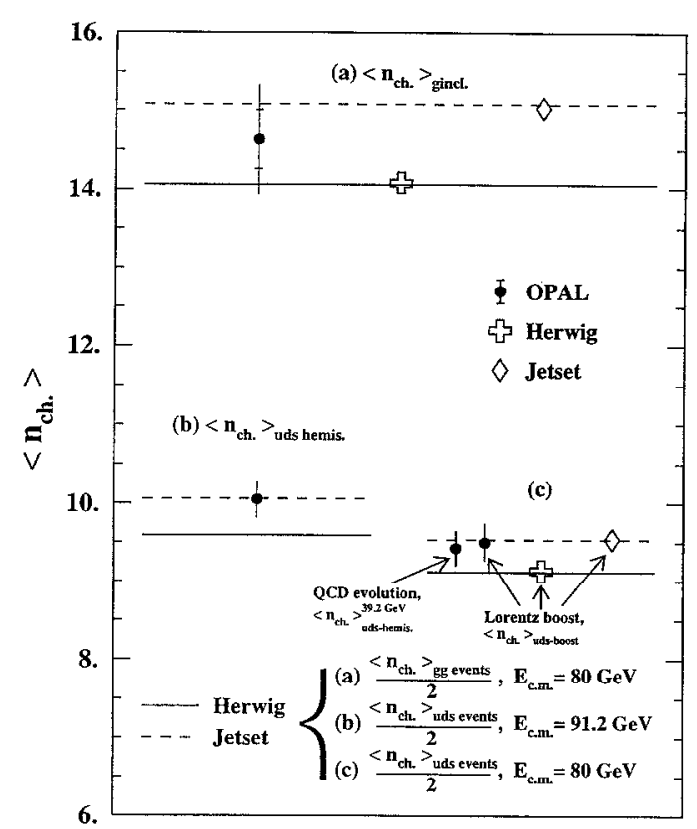

Fig. 3. Corrected results for the menn charged particle multiplicity values of (a) $39.2 \mathrm{GeV}$ gluon jets, (b) $45.6 \mathrm{GeV}$ uds jets, and (c) $39.2 \mathrm{GeV}$ uds jets, in comparison to the generator predictions of the Herwig 5.8 and Jetset 7.4 Monte Carlos. The experimental statistical uncertainties are indicated by the small horizontal bars. The statistical uncertainties are too small to be visible in parts (b) and (c). The statistical uncertainties of the Monte Carlo results are much smaller than those of the data.

results are shown by the solid and dashed horizontal lines in Fig. 3(a). A c.m. energy of $80 \mathrm{GeV}$ is chosen for these gg samples since this is approximately twice the mean energy of $39.2 \mathrm{GeV}$ of the $\mathrm{g}_{\text {incl. }}$ jets. It is seen that the results for the gg and $\mathrm{g}_{\text {incl. samples }}$ agree well, i.e. $\left\langle n_{\mathrm{ch} .}\right\rangle_{\mathrm{g}_{\text {incl. }}} \approx \frac{1}{2}\left\langle n_{\mathrm{ch}}\right\rangle_{\mathrm{gg} \text { events }}$ for both Herwig and Jetset (compare the cross symbol to the solid line and the diamond symbol to the dashed line in Fig. 3(a)). This gives us confidence that the properties of the $g$ incl. sample derived from the $\mathrm{e}^{+} \mathrm{e}^{-}$dalia do indeed correspond closely to those of gg events generated from a color singlet point source, confirming the results of [12]. It further illustrates that the two tagged quark jets used for the identification of the $g_{\text {incl. }}$ sample do not have to be truly collinear for the method to be valid [12].

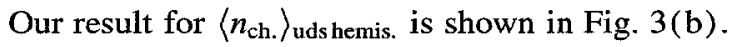
Shown in comparison to this are the results from Herwig and Jetset for half the mean charged particle multiplicity value in uds $q \overline{\mathrm{q}}$ events, $\frac{1}{2}\left\langle n_{\mathrm{ch}} .\right\rangle_{\mathbf{u d s} \text { events }}$ A c.m. energy of $91.2 \mathrm{GeV}$ is chosen in this case to correspond to twice the energy of the measured uds jets. The Jetset result for $\frac{1}{2}\left\langle n_{\text {ch. }}\right\rangle_{\text {uds events }}$ (dashed line) is seen to agree well with the datum. In contrast, the Herwig prediction (solid line) is too low by about one half unit.

Before forming the ratio betwecn the gluon and uds jet measurements, it is necessary to account for the different energies of the two samples: the gluon jets have a mean energy of $39.2 \mathrm{GeV}$ while the uds jets have a mean energy of $45.6 \mathrm{GeV}$ (Sections 3 and 4). To account for the larger energy of the uds jets, we employ the QCD analytic formula for the evolution of the mean event multiplicity in $\mathrm{e}^{+} \mathrm{e}^{-}$annihilations [29]: $\langle n\rangle=N \alpha_{S}^{a} \exp \left(b / \sqrt{\alpha_{S}}\right)$, where $a=$ $0.25+30 n_{f} /\left(27\left(33-2 n_{f}\right)\right), b=3 \sqrt{96 \pi} /(33-$ $2 n_{f}$ ), and $N$ is an energy independent normalization factor. This QCD result is known to describe the energy evolution of the mean charged particle multiplicity in inclusive $\mathrm{e}^{+} \mathrm{e}^{-}$annihilation events with good accuracy [30], using the two loop formula for $\alpha_{S}$. Assuming $n_{f}=5$, the QCD evolution formula predicts the mean multiplicity in $78.4 \mathrm{GeV}$ events to be $(6.2 \pm 0.4) \%$ smaller than in $91.2 \mathrm{GeV}$ events, where the uncertainty results from the maximum variation found by using the jet energies ( 39.2 and $45.6 \mathrm{GeV}$ ) rather than the event energies, $n_{f}=3$ rather than $n_{f}=5$, and varying the value of $\alpha_{S}$ within its allowed range [20]. A difference of $6.1 \%$ is found if the evolution formula is evaluated using the fitted values for $N$ and $\alpha_{S}$ given in [31], which is virtually identical to the difference of $6.2 \%$ quoted above. Applying a multiplicative correction of $0.938 \pm 0.004$ to the $45.6 \mathrm{GeV}$ uds jet measurement presented above yields $\left\langle n_{\text {ch. }}\right\rangle_{\text {uds hemis. }}^{39.2 \mathrm{GeV}}=9.43 \pm 0.06$ (stat.) \pm 0.22 (syst.) for the mean multiplicity of $39.2 \mathrm{GeV}$ uds jets. This result is shown by the solid point labelled "QCD evolution" in Fig. 3(c). The Jetset prediction for the hemisphere multiplicity in $80 \mathrm{GeV}$ uds events, shown by the dashed line, is seen to agree well with this result. The corresponding prediction of Herwig, shown by the solid line, is somewhat lower than the measurement.

As an alternative method to account for the multiplicity difference between the 45.6 and $39.2 \mathrm{GeV}$ uds jets, we employ the following method which is motivated by relativistically covariant models for jet hadronization such as the phenomenologically suc- 
cessful Lund string model [32]. The particles in a uds jet are boosted by a Lorentz transformation along the thrust axis in the direction towards the opposite hemisphere (i.e., towards the hemisphere containing the tagged uds jet). The properties of the boosted jet are then calculated using the boosted energy and momenta of particles which remain in the hemisphere. As a consequence of the boost, the mean opening angle between particles in the jet becomes larger, the energies of the particles become smaller, and particles near the hemisphere boundary may move into the opposite hemisphere and thus out of the jet: the multiplicity of the jet thereby also decreases. Using the Monte Carlo, we find that a boost factor $\beta=0.185$ accurately accounts for the difference in jet properties between 45.6 and $39.2 \mathrm{GeV}$. For example, the cross and diamond symbols in Fig. 3(c) show the mean hemisphere multiplicity in uds events generated at $E_{\text {c.m. }}=91.2 \mathrm{GeV}$ which have been boosted by the factor $\beta=0.185$ in the manner described above. The agreement between the results from the boosted $91 \mathrm{GeV}$ hemispheres and the unboosted $80 \mathrm{GeV}$ ones (the horizontal lines) is essentially perfect for both Herwig and Jetset, establishing the viability of this technique. Applying the boost procedure to the experimental uds jet data yields the result $\left\langle n_{\text {ch. }}\right\rangle_{\text {uds boost }}=9.50 \pm 0.04$ (stat.) $\perp 0.24$ (syst.) for the mean multiplicity of uds jets with an energy of $39.2 \mathrm{GeV}$, which is almost the same as the value of $\left\langle n_{\mathrm{ch}}\right\rangle_{\mathrm{udsh}}^{39.2 \mathrm{GeV}}$ presented in the previous paragraph. This further increases our confidence that our estimate of the mean multiplicity of $39 \mathrm{GeV}$ uds jets is reliable. The result for $\left\langle n_{\mathrm{ch}} .\right\rangle_{\text {uds boost }}$ is shown by the solid point labelled "Lorentz boost" in Fig. 3(c).

Our result for the multiplicity ratio $r_{\text {ch. }}$. between $39 \mathrm{GeV}$ gluon and quark jets is

$$
\begin{aligned}
r_{\text {ch. }} & \equiv \frac{\left\langle n_{\text {ch. }}\right\rangle_{g_{\text {incl. }}}}{\left\langle n_{\text {ch. }}\right\rangle_{\text {uds hemis. }}^{9.2 \mathrm{GeV}}} \\
& =1.552 \pm 0.041 \text { (stat.) } \pm 0.061 \text { (syst.) }
\end{aligned}
$$

where the systematic uncertainty is discussed in Section 8 . Our measurement of $r_{\mathrm{ch}}$. is shown by the solid point in Fig. 2. The experimental statistical uncertainty is indicated by the small horizontal bars. The measured value of $r_{\mathrm{ch}}$, is seen to agree well with Herwig and Jetset at the hadron level and with Herwig at the parton level. Were we to adopt the alternative definition of $r$ mentioned at the end of Section 6 , by subtracting unity from the numerator and denominator before forming the ratio, our result would be $r_{\mathrm{ch}}=$ $1.617 \pm 0.047$ (stat.) \pm 0.068 (syst.) which is not very different from the result given in relation (2). Thus, our result is effectively stable to the definition of $r$.

For jet energies of $39 \mathrm{GeV}$, the n.n.l.o. calculation which incorporates energy conservation [3] predicts values of $r$ between 1.83 (if $n_{f}=3$ and the energy scale of $\alpha_{S}$ is $E_{\text {c.m. }}$ ) and 1.64 (if $n_{f}=5$ and the energy scale is $E_{\text {c.m. }} / 4$ ): this last value is only slightly above the measured result given above in relation (2). The analytic result is valid for quarks and gluons while our measurement refers to charged hadrons. Jetset predicts a hadronization correction for $r_{\text {ch. }}$, defined by the ratio of the parton to hadron level curves in Fig. 2 (for $E_{\text {jet }}=39 \mathrm{GeV}$ ), of 0.91 . The corresponding prediction from Herwig is 1.02. In this sense, the hadronization correction can be estimated to be about unity and to have an uncertainty of about $10 \%$. Given the ambiguities of the energy scale at which to evaluate $\alpha_{S}$, of the number of active flavors $n_{f}$, of the hadronization correction, and due to the approximate nature by which energy-momentum conservation is included, we conclude that the analytic calculation of Dremin, Hwa and Nechitailo [3] is in general agreement with our measurement. In contrast, the analytic results which do not incorporate energy conservation $[1,2]$ are seen to be in clear disagreement with this measurement, even considering the theoretical ambiguities.

\section{Systematic uncertainties}

To evaluate systematic uncertainties, the analysis was repeated with the following changes relative to the standard analysis. The estimated purities of the $\mathrm{g}_{\text {incl. }}$ and uds jet samples did not change appreciably as the analysis conditions were changed unless otherwise noted.

1. Charged tracks alone were used for the data and for the Monte Carlo samples which include detector simulation, rather than charged tracks plus unassociated electromagnetic clusters. The resulting $\mathrm{g}_{\text {incl. }}$ jet sample contained 258 events, $76 \%$ of which were in common with the events of the standard $g_{\text {incl. }}$ sample.

2. Charged tracks and electromagnetic clusters were restricted to the barrel region of the 
detector only, $|\cos (\theta)|<0.70$, rather than $|\cos (\theta)|<0.94$ for the charged tracks and $|\cos (\theta)|<0.98$ for the clusters. Of the 168 $\mathrm{g}_{\text {incl. }}$ jets selected using this condition, $87 \%$ were in common with the standard analysis.

3. The minimum momentum of charged tracks, $p_{\text {min. }}^{\text {ch. }}$ was increascd from $0.10 \mathrm{GcV} / c$ to $0.20 \mathrm{GeV} / c$.

4. The gluon jet selection was performed using the JADE-E0 [33] and cone [34] jet finders to define the tagged quark jets, rather than the $k_{\perp}$ jet finder: 283 and $215 \mathrm{~g}_{\text {incl. jets resulted, respec- }}$ tively, of which $88 \%$ and $80 \%$ were in common with the standard $g_{\text {incl. }}$ jet sample.

5. The gluon jet sample was restricted to events collected within $100 \mathrm{MeV}$ of the $Z^{0}$ peak, resulting in a reduction of the $\mathrm{g}_{\text {incl. }}$ sample to 200 jets.

6. The gluon jet selection was performed with the requirement that the angle between the two jets in the tagged hemisphere be $80^{\circ}$, rather than $50^{\circ}$ : the number of jets in the $g_{\text {incl. }}$ sample decreased to 180 while their estimated purity increased to $92.0 \%$ (the mean gluon jet energy was reduced to $37.4 \mathrm{GeV}$ from $39.2 \mathrm{GeV}$; a multiplicative correction of 1.02, obtained from Jetset by taking the ratio of $\left\langle n_{\mathrm{ch}}\right\rangle$ in gg events generated at $E_{\text {c.m. }}=78.4 \mathrm{GeV}$ to that generated at $74.8 \mathrm{GeV}$, was applied to account for this energy difference).

7. The two tagged quark jets were required to lie within $65^{\circ}$ of the thrust axis, rather than $70^{\circ}$, resulting in a reduction of the $g_{\text {incl. }}$ sample by $10 \%$ (the resulting change in the mean gluon jet energy was only about $0.10 \mathrm{GeV}$ and was ignored).

8. uds jets were identified without imposing any requirement on the $x_{\max }$ values of the tagged jets: the estimated purity of the uds sample dropped to $78.1 \%$ while the size of the sample increased by a factor of about 13 .

9. uds jets were tagged using charged tracks that appeared within a cone of half angle $70^{\circ}$ around the thrust axis, ratber than $40^{\circ}$ : the number of uds jets decreased to 24091 .

10. The method based on a Lorentz boost was used to account for the difference between the uds and $g_{\text {incl. }}$ jet energies, rather than the method based on the QCD evolution formula.
The differences between the standard results and those found using each of these conditions are listed in Table 1. These differences were used to define symmetric systematic uncertainties. For the systematic term involving the jet finder choice (item 4 in the above list), the larger of the differences between the JADE-E0 or cone jet finders with respect to the standard results was used to define the systematic uncertainty. The uncertainties associated with each item were added in quadrature to define the total systematic uncertainties, given in the last row of Table 1. The systematic uncertainties for the $39.2 \mathrm{GeV}$ uds jet measurement were derived from those evaluated for the $45.6 \mathrm{GeV}$ uds jet measurement using the multiplicative correction of 0.938 obtained from the QCD evolution formula.

From Table 1, it is seen that the largest systematic term for the gluon jet multiplicity $\left\langle n_{\mathrm{ch}}\right\rangle_{\mathrm{g}}$ incl. and for the multiplicity ratio $r_{\text {ch. }}$ is from the difference between the charged only and standard results. In contrast, there is only a small change in the results if the JADE-E0 or cone jet finders are used to tag quark jets for the $\mathrm{g}_{\text {incl. }}$ identification rather than the $k_{\perp}$ jet finder. This emphasizes that our results are only weakly dependent on a jet finding algorithm. For the uds jet multiplicity, $\left\langle n_{\mathrm{ch} .}\right\rangle_{\text {uds hemis. }}$, the largest systematic term is associated with the $x_{\max }$ cut.

As an additional check, the analysis was repeated with the requirement that there be at least one significant track in the secondary vertices used to tag quark jets for the gluon jet selection, rather than at least two such tracks: the number of jets in the $g_{\text {incl. }}$ sample increased to 647 while their estimated purity dropped to $61.8 \%$. The results remained consistent with the standard results: for example, we obtained $r_{\text {ch. }}=1.581 \pm$ 0.031 (stat.) using this variation in the analysis. The results of this change were not included in the total systematic uncertainties since item 5 in the above list already assesses the consequence of a large change in the estimated gluon jet purity. A last check was performed by comparing the results obtained using Jetset 7.3 to define the detector corrections with those obtained using Jetset 7.4, rather than using the combined Jetset sample. There are significant differences between these two Jetset versions, as implemented by OPAL, for $b$ hadron properties such as mean energy and decay multiplicity [7]. The results obtained using the two Jetset versions to define the detector corrections were virtually identical, establishing that imper- 
Table 1

Difference between the results of the standard analysis and those found by repeating the analysis with the changes listed.

\begin{tabular}{|c|c|c|c|c|}
\hline & $\left\langle n_{\mathrm{ch} .}\right\rangle_{\mathrm{g}}$ incl. & $\left\langle n_{\mathrm{ch}}\right\rangle_{\text {uds hemis. }}$ & $\left\langle n_{\text {ch. }}\right\rangle_{\text {uds hemis. }}^{39.2 \mathrm{GeV}}$ & $r_{\text {ch. }}$ \\
\hline 1. Charged tracks only & -0.39 & -0.01 & -0.01 & -0.040 \\
\hline 2. $\left|\cos \left(\theta_{\text {particle }}\right)\right|<0.70$ & -0.28 & -0.14 & -0.13 & +0.007 \\
\hline 3. $p_{\min }^{\text {ch }}>0.20 \mathrm{GeV} / c$ & -0.14 & -0.02 & -0.02 & -0.011 \\
\hline 4. JADE-E0 jet finder & -0.22 & - & - & -0.023 \\
\hline ( Cone jet finder & -0.16 & - - & -- & -0.017 ) \\
\hline 5. On-peak data only & -0.19 & - & - & -0.020 \\
\hline 6. $\theta_{\text {jet } \mathrm{a}-\text { jet } \mathrm{b}}>80^{\circ}$ & +0.13 & - & - & +0.014 \\
\hline 7. $\theta_{\text {jet a }}-$ thrust,$\theta_{\text {jet b-thrust }}<65^{\circ}$ & +0.03 & - & - & +0.003 \\
\hline 8. No $x_{\max }$ cut & - & +0.18 & +0.17 & -0.027 \\
\hline 9. $70^{\circ}$ cone & - & +0.03 & +0.03 & -0.005 \\
\hline 10. Lorentz boost method & - & - & - & -0.010 \\
\hline Total systematic uncertainty & 0.59 & 0.23 & 0.22 & 0.061 \\
\hline
\end{tabular}

fect knowledge of the $b$ hadron production and decay mechanisms does not have a significant influence on our results.

\section{Summary and conclusions}

In this paper, we have presented a quantitative test of analytic predictions for the ratio of the mean particle multiplicity between gluon and quark jets. The technique that allows this test is the identification of gluon jets in an inclusive manner, by all the particles observed in the event hemisphere opposite to a hemisphere containing a tagged quark and antiquark jet [12]. The resulting definition is in close correspondence to the definition of gluon jets in analytic calculations, for the first time in the analysis of high energy $\mathrm{e}^{+} \mathrm{e}^{-}$data. In our study, the gluon jet measurement, valid for $39 \mathrm{GeV}$ jets, is compared to the corresponding measurement from light quark (uds) jets, which has also been defined inclusively.

Our result for the ratio $r_{\text {ch., }}$, the mean charged particle multiplicity of gluon jets divided by the corresponding value for uds quark jets, is $r_{\mathrm{ch} .}=1.552 \pm$ 0.041 (stat.) \pm 0.061 (syst.). This result is substantially smaller than the predictions of analytic calculations which do not include energy conservation in the parton branchings. Using the Herwig and Jetset Monte Carlos, we find that hadronization uncertainties are unlikely to explain the discrepancy betwcen our result and these analytic predictions. A recent analytic cal- culation [3] which incorporates approximate energymomentum conservation predicts a parton level multiplicity difference between $39 \mathrm{GeV}$ gluon and quark jets in the range from about 1.64 to 1.83 , depending on the choice for the energy scale $Q$ at which the strong coupling constant $\alpha_{S}(Q)$ is evaluated and on the number of active quark flavors, $n_{f}$. This latter prediction is in overall agreement with our measurement, given the uncertainties due to the approximate nature of energy-momentum conservation in the calculation, missing higher order terms, the energy scale, $n_{f}$, and hadronization.

\section{Acknowledgements}

We thank Igor Dremin, Rudy Hwa, Mike Seymour, Torbjörn Sjöstrand and Bryan Webber for helpful comments.

We particularly wish to thank the SL Division for the efficient operation of the LEP accelerator and for their continuing close cooperation with our experimental group. In addition to the support staff at our own institutions we are pleased to acknowledge the Department of Energy, USA, National Science Foundation, USA, Particle Physics and Astronomy Research Council, UK,

Natural Sciences and Engineering Research Council, Canada,

Israel Ministry of Science, 
Israel Science Foundation, administered by the Israel Academy of Science and Humanities,

Minerva Gesellschaft,

Japanese Ministry of Education, Science and Culture (the Monbusho) and a grant under the Monbusho International Science Research Program,

German Israeli Bi-national Science Foundation (GIF),

Direction des Sciences de la Matière du Commissariat à l'Energie Atomique, France,

Bundesministerium für Bildung, Wissenschaft, Forschung und Technologie, Germany, National Research Council of Canada, Hungarian Foundation for Scientific Research, OTKA T-016660, and OTKA F-015089.

\section{References}

[1] S.J. Brodsky and J. Gunion, Phys. Rev. Lett. 37 (1976) 402; K. Konishi, A. Ukawa and G. Veneziano, Phys. Lett. B 78 (1978) 243.

[2] A. H. Mueller, Nucl. Phys. B 241 (1984) 141; J.B. Gaffney and A.H. Mueller, Nucl. Phys. B 250 (1985) 109;

E.D. Malaza and B.R. Webber, Nucl. Phys. B 267 (1986) 702.

[3] I.M. Dremin and R.C. Hwa, Phys. Lett. B 324 (1994) 477; I.M. Dremin and V.A. Nechitailo, Modern Phys. Lett. A 9 (1994) 1471.

[4] OPAL Collaboration, G. Alexander et al., Phys. Lett. B 265 (1991) 462.

[5] OPAL Collaboration, P.D. Acton et al., Z. Phys. C 58 (1993) 387.

[6] OPAL Collaboration, R. Akers et al., Z. Phys. C 68 (1995) 179.

[7] OPAL Collaboration, G. Alexander et al., Z. Phys. C 69 (1996) 543.

[8] DELPHI Collaboration, P. Abreu et al., Z. Phys. C 70 (1996) 179.

[9] ALEPH Collaboration, D. Buskulic et al., Phys. Lett. B 346 (1995) 389.

[10] ALEPH Collaboration, D. Buskulic et al., CERN-PPE/95184.

[11] For recent reviews, see: I.G. Knowles et al., Physics at LEP 2, Vol. 2, CERN 96-01, eds. G. Altarelli and F. Zwirner; J.W. Gary, Proceedings of the XXV International Symposium on Multiparticle Dynamics, Stará Lesná, Sloyakia, September 12-16, 1995.

[12] J.W. Gary, Phys. Rev. D 49 (1994) 4503.

[13] OPAL Collaboration, K. Ahmet et al., Nucl. Instr. and Meth. A 305 (1991) 275;

P. Allport et al., Nucl. Instr. and Meth. A 324 (1993) 34;

P. Allport et al., Nucl. Instr. and Meth. A 346 (1994) 476.

[11] S. Brandt et al., Phys. Lett. 12 (1961) 57;

E. Fahri, Phys. Rev. Lett. 39 (1977) 1587.

[15] S. Catani et al., Phys. Lett. B 269 (1991) 432.

[16] T. Sjöstrand, Comp. Pliys. Comm. 82 (1994) 74; T. Sjöstrand, CERN-TH.7112/93 (revised August 1995).

[17] J. Allison et al., Nucl. Instr. and Meth. A 317 (1992) 47.

[18] See, for example: V. Khoze, in: Proc. Int. Symp. Lepton Photon Interactions, Stanford 1989, ed. M. Riordan (World Scientific, Singapore) 1990.

[19] OPAL Collaboration, P.D. Acton et ai., Z. Phys. C 58 (1993) 523.

[20] L. Montanet et al., Phys. Rev. D 50 (1994) 1173 and 1995 off-year partial update for the 1996 edition available on the PDG WWW pages (URL: http://pdg.lbl.gov/).

[21] Z. Kunszt, P. Nason et al., Z Physics at LEP 1, Vol. 1, CERN 89-08, eds. G. Altarelli, R. Kleiss and C. Verzegnassi, Geneva 1989.

[22] W.J. Marciano, Phys. Rev. D 29 (1984) 580.

[23] W. Bernreuther, Annals of Physics 151 (1983) 127.

[24] G. Marchesini, B.R. Webber et al., Comp. Phys. Comm. 67 (1992) 465.

[25] OPAL Collaboration, R. Akers et al., Z. Phys. C 68 (1995) 203.

[26] Torbjörn Sjöstrand, private communication.

[27] Igor Dremin, private communication.

[28] OPAL Collaboration, R. Akers et al., Phys. Lett. B 352 (1995) 176.

[29] A.H. Mueller, Nucl. Phys. B 213 (1983) 85; A.H. Mueller, Nucl. Phys. B 228 (1983) 351;

B.R. Webber, Phys. Lett. B 143 (1984) 501.

[30] DELPHI Collaboration, P. Abreu et al., Z. Phys. C 50 (1991) 185;

ALEPH Collaboration, D. Decamp et al., Phys. Lett. B 273 (1991) 181;

OPAL Collaboration, P.D. Acton et al., Z. Phys. C 53 (1992) 539.

[31] OPAL Collaboration, G. Alexander et al., CERN-PPE/96047 , in print in $\mathrm{Z}$. Phys. C.

[32] B. Andersson et al., Phys. Rep. 97 (1983) 31.

[33] JADE Collaboration, W. Bartel et al., Z. Phys. C 33 (1986) 23.

[34] OPAL Collaboration, R. Akers et al., Z. Phys. C 63 (1994) 197. 